\title{
THE USE OF INTERMITTENT NORMOBARIC HYPOXIA IN CHILDREN'S NEUROLOGY (REVIEW)
}

\author{
Lyubov Lisukha \\ Department of clinical physiology connective tissues \\ O. O. Bogomolets Institute of Physiology, NAS of Ukraine \\ 4 Bogomoltsa str., Kyiv, Ukraine, 01024 \\ lyubovlisukha@gmail.com
}

\begin{abstract}
The present review summarizes the data about the use of intermittent normobaric hypoxia (INH) in the treatment and prevention of both acute and chronic diseases of the nervous system in children. The INH method is used in pregnant women with fetoplacental insufficiency, anemia and for mental disorders correction. The INH efficiency in relation to such pathology as cerebral palsy, epilepsy, enuresis, sleep disturbances, autonomic dysfunction is proved. The age dependence of bioelectric activity of the brain in practically healthy persons (age from 8 to 21 years) on hypoxic factor and the most sensitive period of age from 13 to 16 years is shown. The results the own studies have shown that the children which are born and live in radioactive contaminated areas (RCA), the course of INH sessions led to the restoration of somatic vegetative status: activation of higher vegetative centers, optimization of sympathetic and parasympathetic links; positive influence on the activity of the cardiovascular system; ventilatory capacity of lungs; blood parameters.
\end{abstract}

Keywords: intermittent normobaric hypoxia, neurology, children.

\section{Introduction}

A normobaric hypoxic test using gas mixture containing $10 \%$ oxygen and $90 \%$ nitrogen for 20 minutes was used for the first time in 1939 by P. Ber and R. Levy to estimate the status of coronary blood flow [1]. In 1970, R. B. Strylkov formulated the concept of the possibility of protection from ionizing radiation by hypoxic gas mixture (HGM) with an oxygen content of $10.0 \pm 1.0 \%$ [2]. In 1980, he and his coworkers offered to replace the mountain and hypobaric chamber training by HGM respiration (with low oxygen content at normal atmospheric pressure). Studies under the guidance of O. Y. Chizhov [3] and Yu. M. Karash [4] contributed to the substantiation and implementation of the method in medical practice. It is known as intermittent normobaric hypoxia (INH) [5] or instrumental orotherapy [6].

According to the definition of "intermittent normobaric hypoxytherapy" (INH), a "mountain air" is a non-medicinal method for increasing the non-specific resistance of the organism to the damaging factors of external and internal environment, which ensures the development of the dosage in the depth and time of hypoxia in human's body when breathing by gas mixtures with a low oxygen content" [7].

The use of the course of INH sessions increases the nonspecific resistance of the body. In the process of adaptation to INH, the oxygen function of the blood, microcirculation, efficiency of the cardiopulmonary resuscitation system, activity of the immune, antioxidant and endocrine systems are improved; neurohumoral response to psychological trauma is decreased; intoxication is reduced; resistance to various factors including to ionizing radiation is increased.

\section{Aim of Research}

To analyze the tendencies to the use of INH method in children having nervous system pathology.

\section{Hypoxiotherapy in the treatment and prevention of psychological and neurological disor- ders in children}

The study and analysis of the national and foreign literature sources shows that the mechanisms of INH influence on the central nervous system (CNS) are widely studied both in models and in clinical practice. There are several hundred of such studies. In review articles, in particular, in 
recent years, the relevance of this method and its use as a preconditioning [8] and by courses: normobaric hypoxic training in practically healthy people, and hypoxitherapy in the pathology of the nervous system has been highlighted. Thus, in [9] different known mechanisms of adaptation to the the hypoxic factor of the brain are described. It is known that the nervous system is most susceptible to hypoxia. The effect of the latter on the central nervous system, hemodynamics is shown, and improvement of cerebral circulation is an important adaptive effect [9]. Other authors [10] consider pathogenetic bases of the INH method as a result of compensatory and adaptive reactions in human's body and changes in various organs and systems under the INH influence. Researchers point to their own experience of using of the given method in children with cerebral palsy and epilepsy. The review [11] both the relevance and the effectiveness of the INH use in the rehabilitation of patients with neurological and somatic profile was analyzed, which occurs as a result of the activation of adaptive, immunological, biochemical and hemodynamic sanogenesis mechanisms. Concerning the improvement of the mental capacity of the brain, the work [12] shows that after the INH conditioned reflex activity and functional mobility of the nervous processes have increased in practically healthy persons aged 19 to 21 years. In other studies [13] in the elderly people (from 60 to 74 years), on the contrary, reduction of conditioned reflex activity was registered, which may be due to more severe arterial hypoxemia (as compared to young people aged 20 to 29 years) as a result of lower oxygen saturation in the lungs during hypoxic effects. Positive effect of INH as monotherapy in patients with endogenous depression and its efficacy up to $71 \%$, and at post-schizophrenic depression to $57 \%$ is known [14]. The author [15] conducted a review of the PubMed literature database concerning the study and the use of hypoxia in relation to mental diseases.

Nowadays, there are dozens of works about the application of this method in nervous system pathology in children. The majority of studies have been carried out by national scientists and in the NIS countries.

The development of the child's nervous system depends on many factors, including mother's health. Therefore, INH is used to prepare women with negative obstetric history, chronic somatic diseases few months before pregnancy, to prevent abnormalities in the antenatal development of the fetus $[16,17]$. In pregnant women it is used from the first trimester for prevention of toxicosis, anemia (pregnant women), fetoplacental insufficiency, hypoxia and hypotrophy (of the fetus) [18, 19]. The scientists have found that during the development of neurohumoral regulation of the fetus during the second trimester of pregnancy, an increased anxiety, depression, stress that is accompanied by hormonal changes in blood may happen, affecting the development of the child's nervous system [20,21]. Experimental and epidemiological studies indicate that in future it rather difficult for newborns to adapt to the environment, and negative effects in physical, neurological and mental development may occur $[22,23]$. Significant hypoxia is a trigger for an abnormal brain development, which leads to mental disorders in adolescents and early adulthood [24].

The use of INH with an oxygen content of $10 \%$ in pregnant normalizes uteroplacental circulation due to adaptive structural changes that allows maintaining homeostasis in the motherplacenta-fetus system under hypoxia conditions [25]. Besides, therapeutic effect, which is manifested by increased reserve of the cardiopulmonary system, was found; psycho-emotional stress and autonomic manifestations decrease, and blood pressure, estrogen content and progesterone acid-base state are normalized. The researchers [26] have shown that long-term adaptation to hypoxia, the expression of the NO-synthase gene increase, that promotes endogenous NO production and creates a $\mathrm{NO}$ depot in vessel's endothelium. In future, this can prevent blood pressure increase. In the studies [27] INH (with an oxygen content of 13-10\%) was used in pregnant women (the period of pregnancy 20-32 weeks) with varying degrees of anemia. It is shown that after INH the parameters of erythrocytic chain, iron metabolism are improved; the acid-base state and the content of blood electrolytes are normalized; a therapeutic effect was obtained (as in [25]). Normobaric hypoxic therapy can be used to prevent mental diseases: one course before pregnancy and one or two (during pregnancy) after the $16^{\text {th }}$ week [7]. It has been found that children born to mothers who had INH sessions had a high Apgar score, larger body weight and body length. They had significantly fewer cases of perinatal lesion of the central nervous system, cerebrovascular disorders, and encephalopathy [25]. 
In pediatric neurology, the method of normobaric hypoxia is used, first of all, in child cerebral palsy. After a course of INH sessions using HGM $12 \%$ of $\mathrm{O}_{2}$ in nitrogen, a tendency to improve coordination and chaotic movements decrease children aged 3 to 18 years, is observed in $90 \%$ of patients, also the volume of active (89\%) and passive (in $87 \%$ ) movements in joints increases, muscular tone in the limbs decreases, the foot support becomes more confident and the clones in the lower extremities decrease [28], the complete or partial overcoming of pathological synergies is recorded [29]. Researchers explain this fact by changing the activity of GABA-ergic and serotonergic systems due to the INH effects, since they provide the processes of inhibition and coordination of movements [30]. According to foreign authors, it confirms the hypothesis concerning the fact that anaerobic potential is an important element in physical role of routine daily activities in people with child cerebral palsy [31].

In other studies, a comprehensive examination of 87 patients aged from 8.5 months to 12 years, have shown that in $85 \%$ of this patients with the given pathology blood flow of the brain (according to transcranial Doppler) and ophthalmoscopic pattern (32\%) are improved. The positive change in the electroencephalograms is noted, in particular epileptiform manifestations decrease: the density of alpha rhythm power increases by $23 \%$, beta - by $26 \%$, delta and theta rhythm are reduced by $35 \%$. Improvement of local blood supply to the brain, ophthalmoscopic picture and bioelectric activity markers are considered by the authors as adaptive reaction to the course of hypoxia therapy sessions [29]. In addition, fatigue, enuresis, anxiety levels decrease, sleep improves, therefore, a positive dynamics of somatic and psychophysiological conditions affecting the quality of life are observed [32].

Research concerning the study of epileptic disease in children and adolescents show that its course is accompanied by free radical processes activation and inhibition of antioxidant defense in blood and brain. In an experiment in rats, it was shown that hypoxic effect exactly (HGM respiration with oxygen content of 10-12\%) activates peroxide-oxidative and antioxidant processes in the blood [33].

In adolescent patients with epilepsy, under the influence of INH both the minimum and maximum concentration of antiepileptic drugs decreased. At the same time, after the course of sessions, the frequency, duration and severity of attacks decreased. According to the researchers, this property is connected to the activation of energy processes, which confirms the efficiency of the therapy. The study of bioelectric activity of the brain showed that after the first session, the power in the slow waves range increases and epileptic activity decreases. Scientists believe that such positive effect may be explained by the involvement of the main part of neurons in generation of non-epileptic forms of activity and points to adaptive processes in brain in response to the shortterm effect of INH. The change of the electroencephalogram in the a- and $\beta$-frequency range gives reasons to affirm about the neurophysiological normalization of bioelectric activity of brain and correlates with the resistance of the therapeutic effect [34].

Recent studies in area of brain bioelectrical activity in practically healthy children and adolescents under conditions of short-term hypoxic influence has an age-dependent dependence of the organism on hypoxia: at the age of 8-12 years and 17-21 years the activity of the cerebral cortex is recorded, at the age of 13-16 years, the activity of the subcortical structures is found. In all patients (from 8 to 21 years old) the index and amplitude of alpha, theta, and delta waves is increased. It should be noted that the amplitude of fast alpha activity (aged 13 to 16 years) is increasing, which according to researchers points to an increased sensitivity of this age group to the effect of shortterm hypoxia. Under conditions of hypoxia, the rates of excitatory and inhibitory tests tended to decrease, and movements' coordination during the Thorndike labyrinth probably got worse. More mistakes were recorded in children aged 13 to 16 [35].

41 children (aged 8 to 14 years) with vegetative dysfunctions were examined. After the hypoxic test for 15 minutes $\left(14 \% \mathrm{O}_{2}\right)$, normal blood pressure (BP), heart rate (heart rate) were normalized; according to the analysis of the heart rate variability, the stress index decreased [36]. In other studies, 166 patients aged 12 to 15 years with arterial hypertension were examined, 91 of them had normobaric hypoxic therapy prior to baseline therapy. After the course of the INH sessions, the average daily blood pressure indices were reduced, and stabilization or reduction of the hypertension 
time index allows asserting about a more favorable prognosis compared with control. Besides, complaints of headaches, associated vegetative manifestations, and emotional stress decrease [37]. The authors testify that in practically healthy children the course of INH sessions promotes the change of the initial vegetative tone to atonia [38]. In children aged 10 to 13 years living in RCA and having vegetative disorders, normobaric interval hypoxic training increase the parameters of the Stange test, reduce the manifestations of vegetative homeostasis towards sympathicotonia and increase the positive effect of balneotherapy [39]. The basis of this is the physiological effect of INH, namely the known mechanisms of hypoxic training are started. In particular, collateral circulation is activated, blood rheological properties change, nerve-reflex regulation of the vascular tone improves, and venous congestive events in the brain decrease, the cardiac muscle activity and cardiorespiratory system function improve, the oxygen delivery to the periphery increases.

In 35 preschool children (5 to 6.5 years) having neuropsychological disorders, the use of INH helped to change the emotional state, alignment of brain asymmetry with activation of the left hemisphere and the formation of individual style of activity [40]. Some researchers note that the use of the INH sessions course (within 2 weeks) in patients of middle and high school age (12 to 17 years) with intellectual disabilities helped to improve psycho-emotional stability, reduce anxiety, increase mobility of nervous processes, which indicates an improvement in the perception and processing of information [41].

It is known that even after single hypoxic effects in brain structures, expression of NCFI-A, c-jin, jinB, and c-foc, playing a significant role in neuronal plasticity processes, training, survival/ death of neurons, is increased [42]. It has been determined that the neuroprotective effect of hypoxic preconditioning is the result of stimulation of erythropoietin receptors [43] and increased activity of PI3-Akt [44] and ERK1/2 [45]. In recent years, scientists have shown a positive effect of hypoxia on the model of mitochondrial diseases. Thus, in rats with a deficiency of the Ndufs 4 gene (the gene encoding protein for mitochondria (complex I)) that were in cells with $11 \%$ of $\mathrm{O}_{2}$, the life duration was 270 days, and in animals at $21 \% \mathrm{O}_{2}-60$ days. The mutation of the Ndufs 4 gene is observed in some patients with Lee's syndrome [46].

\section{Results of research}

The effect of INH on the somatic vegetative parameters in children living in RCA, as a result of the Chernobyl accident (1986) was studied. 100 patients aged 6 to 17 years had the course of INH sessions $\left(12 \% \mathrm{O}_{2}\right)$. The results indicate higher vegetative centers activation, sympathetic and parasympathetic links and optimization of their correlation, positive effects on the cardiovascular system activity, pulmonary ventilation, and blood parameters [47, 48]. Increasing the L-arginine content by $9.1 \%$ in serum, under the conditions of the absence of significant changes in stable metabolites, NO is considered as a possible mechanism of INH action, which temporarily limits the use of this amino acid and accumulates it for more intensive use in future [49].

Reduction of latent periods of complex visual motor reaction characterizes the speed of visual information cognitive processing and the formation of an adequate visual motor response. In our research, it has been shown that in children living in RCA, the latent periods reduction allows to assert about a certain general activation of the nervous system.

To sum up the literature data and the results of our own studies concerning the changes of autonomic nervous system, it can be noted that the improvement of the somatic vegetative status of the RCA inhabitants is connected with the influence of HGM through impulses from chemoreceptors by afferent nerve fibers to medulla oblongata autonomic centers, hypothalamus and cerebral cortex. As a result, a certain cardiorespiratory relaxation and optimization of sympathetic and parasympathetic effects on the cardiovascular system and external respiration can be achieved. The obtained results of our studies also testify this. It has been shown that INH contributes to accelerating ${ }^{137} \mathrm{Cs}$ excretion from human's body. It improves the general health of children by reducing anxiety, increasing tolerance to psychoemotional and physical activity, and also improving sleep, reducing headaches and period of restitution during active clinoorthostatic testing [50]. 


\section{Conclusion}

1. INH sessions can be useful for women planning pregnancy and living in either environmentally unfriendly areas or radioactive contaminated areas, as well as for women having negative obstetric history and chronic somatic diseases. This method can be used by pregnant women having fetoplacental insufficiency, anemia, for correction of mental disorders.

2. INH is used for prevention, treatment and rehabilitation of both chronic and newly diagnosed nervous system diseases in children.

3. The short-term hypoxic factor influence on the bioelectric activity of the brain of practically healthy children and adolescents is age-dependent. People aged 13 to 16 years are the most susceptible to hypoxia.

4. INH of the sanogenic level improves the somatic vegetative status and accelerates ${ }^{137} \mathrm{Cs}$ excretion in children born and living in radioactive contaminated areas.

5. There is a necessity to study the use of INH method in children having nervous system pathology and mental development disorders, as well as to improve mental abilities. This is a prospect of the future research.

\section{References}

[1] Levy, R. L., Williams, N. E., Bruenn, H. G., Carr, H. A. (1941). The "anoxemia test" in the diagnosis of coronary insufficiency. American Heart Journal, 21 (5), 634-656. doi: 10.1016/s0002-8703(41)90727-5

[2] Strelkov, R. B. (1971). The method of protecting mammals from the effects of ionizing radiation. Author's certificate No. 389549 (USSR).

[3] Chizhov, A. Ya. (1992). A way to increase the compensatory capabilities of the body. Author's certificate No. 950406. Bulletin of Inventions, 2, 33-34.

[4] Karash, Yu. M., Strelkov, R. B., Chizhov, A. Ya. (1988). Normobaric hypoxia in treatment, prevention and rehabilitation. Moscow: Medicine, 351.

[5] Strelkov, R. B., Karash, Yu. M., Chizhov, A. Ya. et. al. (1985). The method of increasing nonspecific resistance of the organism with the help of normobaric hypoxic stimulation. Moscow, 10.

[6] Berezovskiy, V. A., Horban, Y. M., Levashov, M. I., Sutkovsky, A. D. (2000). Technology body resistance increase using hypoxytherapy. Kyiv, 24.

[7] Streilkov, R. B., Chizhov, A. Y. (2001). Intermittent normobaric hypoxia: prevention, treatment, rehabilitation. Ekaterinburg: Ural worker, 400.

[8] Maslov, L. N., Lishmanov, Yu. B., Emelianova, T. V. et. al. (2011). Hypoxic preconditioning as novel approach to prophylaxis of ischemic end reperfusion damage of brain and heart. Angiology and Vascular Surgery, 17 (3), 27-36.

[9] Solkin, A. A., Belyavsky, N. N., Kuznetsov, V. I., Nikolaev, A. G. (2012). The basic mechanisms of formation of protection of the brain during adaptation to hypoxia. Vestnik of the VSM, 11 (1), 6-14.

[10] Shalkevich, L. V., Kulikova, V. V. (2014). Hypoxic adaptation as a method of treatment of neurological diseases. Ukrainian Medical Almanac, 17 (2), 148-151.

[11] Gainetditov, A. R., Khuzina, G. R., Mukhametshina, G. A. (2015). The use of dosed normobaric gipokskoy therapy in the medical rehabilitation of patients with neurological and cooperative profile. Journal of International Medicine, 2 (13), 157-164.

[12] Volobuev, M. I. (1997). Investigation of the influence of breathing sessions artificial mountain air breathing session on sensomotoric cadet's reactivity. Problems of military health care and its reform path. Kyiv: Ukrain military medical acad., 548-550.

[13] Korkushko, O. V., Osmak, E. D. (2013). Age-related characteristics of cerebral cicculation and mental performance in hypoxia at aged healthy people with different types of aging - physiological and accelerated (erly). Csrculation and hemostasis, 2, 7-26.

[14] Karimulaev, I. A., Kalinin, A. V., Maslov, S. N. (2003). Clinical efficiency of the method of adaptation to periodic normobaric hypoxia (APNG) in the treatment of postpsychotic depression in patients with schizophrenia. Social and Clinical Psychiatry, 13 (4), 37-41.

[15] Basovich, S. N. Haypoxia in genesis and therapy of mental deviations: a review (2011). Bulletin of the International Academy of Sciences, 1, 1-12. 
[16] Radetskaya, L., Kuznetsov, V. I., Suprun, L. Y. (2000). Clinical efficiency of application interval normobaric hypoxic therapy in complex treatment of patients with endometriosis. Hypoxia Medical Journal, $1(2), 17-21$.

[17] Kolchinskaya, A. Z., Tsyganov, T. N., Ostapenko, L. A. (2003). Normrbaric interval hypoxic training in medicine and sport. Moscow: Medicine, 408.

[18] Berezovsky, V. A., Yatsenko, K. V. (2010). Instrumental orotherapy in obstetrics and gynecology practice. Kyiv, 21.

[19] Rogozhina, I. E., Makhova, G. E., Prodanova, E. V. (2010). Prospects of application of normobaric hypoxic interval training in treatment of placental insufficiency (literature review). Saratov Journal of Medical Scientific Research, 6 (3), 543-549.

[20] Janus, L. (2013). Sustained effect of prenatal experience. Kyiv: CPU «Kopprit», 290.

[21] Huizink, A. C., Mulder, E. J. H., Buitelaar, J. K. (2004). Prenatal Stress and Risk for Psychopathology: Specific Effects or Induction of General Susceptibility? Psychological Bulletin, 130 (1), 115-142. doi: 10.1037/0033-2909.130.1.115

[22] Rice, F., Jones, I., Thapar, A. (2007). The impact of gestational stress and prenatal growth on emotional problems in offspring: a review. Acta Psychiatrica Scandinavica, 115 (3), 171-183. doi: 10.1111/j.16000447.2006.00895.x

[23] Syusyuka, V. G., Kotlova, Yu. V. (2014). Estimation of influence of medical and psychological correction and sanatorium rehabilitation of pregnant women program on state of newborns and course of their early neonatal period. Sovremennaya pediatriya, 8 (64), 31-35.

[24] Kalus, P., Falkai, P., Heinz, A. (2008). Strukturelle und funktionelle Hirnveränderungen bei schizophrenen Psychosen. Der Nervenarzt, 79 (3), 275-287. doi: 10.1007/s00115-008-2414-5

[25] Tsiganova, T. N., Strelkova, R. B. (2005). Using of normobaric interval (intermittent) hypoxic training to improve the body condition of pregnant women with abnormal pregnancy. Intermittent normobaric hypoxic. Moscow: Paper Gallery, 4, 226.

[26] Bloshchinskaya, I. A. (2003). Functional condition of the vascular endothelium and microcirculatory disturbances in pregnancy complicated by gestosis, and the impact on them of normobaric hypoxic therapy. Khabarovsk, 232.

[27] Vladimirov, O. A., Vladimirova, N. I., Hrydko, L. A. (2010). Increased functional reserves of the body in pregnant women with anemia by means of intermittent normobaric hypoxic stimulation. Probl Phys Educ Sport, 6, 33-35.

[28] Dyakova, E. V. (2005). Normobaric hypoxic therapy in the rehabilitation of various forms of cerebral paralysis in children. Moscow: Medicine, 84.

[29] Yatsenko, K. V., Berezovsky, V. A. (2012). Use of intermittent normobaric hypoxia in combination therapy of infantile cerebral paralysis. Intern Neurological Journal, 1 (47), 51-56.

[30] Hankins, G. D. V., Speer, M. (2003). Defining the Pathogenesis and Pathophysiology of Neonatal Encephalopathy and Cerebral Palsy. Obstetrics \& Gynecology, 102 (3), 628-636. doi: 10.1097/00006250200309000-00036

[31] Dallmeijer, A. J., Balemans, A. C., Wely, Van, L., Scholtes, V. A., Brehm M. A. et al. (2012). Anaerobic capacity in children with cerebral palsy. Intern Neurological Journal, 1 (47), 26-27.

[32] Nasar, O. V. (2011). Effct of normobaric hypoxic training on quality of life children with cerebral palsy. Sovremennaya pediatriya, 5 (39), 100-102.

[33] Sutkovoy, D. A., Laponogov, O. O., Kevorskov, G. A. et. al. (2005). The changes of free-radical activity in children and treenagers suffering from epilepsy and the rehabilitation normobaric hypoxytherapy influence on pro-and atioxidative status. Ukrainian Neurosurgical Journal, 2, 34-39.

[34] Starykh, E. V. (2003). Electroencephalographic control over efficacy of hypoxytherapy as anadjiwant treatment of epilepsy. J Neurol Psychiat., 7, 27-30.

[35] Borukaeva, I. K., Abazova, Z. K., Kumykov, V. K. (2014). The effect of intrermittent hypoxia on bioelectric activity of the brain in children adolescents and youths. Fundamental research, 4 (3), 466-471.

[36] Tyurin, I. A., Korovin, O. A., Kontemirova, M. G., Anokhin, A. I. (1999). Effect of short-term hypoxic load on the autonomic regulation and hemodynamic parameters in children with vegetative dystonia syndrome. Her Russ Univer of Peopl Friendship. Series:»Medicine», 2, 110-114. 
[37] Vlasenko, A. V., Dolgikh, V. V., Richokova, L. V., Bugun, O. V. (2007). The influence of normobaric hypoxic therapy on emotional state of children and teenagers with arterial hypertension. Bull ESSC SB RAMS, 3 (55), 29-31.

[38] Korneeva, I. T., Polyacov, S. D., Hodarev, S. V., Tertyshnaja, Ye. S. (2010). Training process correction of young football players with application of interval hypoxic training. Med Bull North Caucas., $3,110-111$.

[39] Starodub, A. G., Barylyac, L. G., Korolyshyn, T. A., Popovich, I. L. (2012). Appication of interval normobaric hypoxic training for amelioration influence balneotherapeutic complex spa Truscavets on resistence to hypoxia and the autonomic nervous system. Med Hydrol Rehabil., 10 (2), 87-91.

[40] Syazina, N. Y., Frantasova, E. (2014). The Role of Normobaric Hypoxic Therapy in the Formation of an Adequate Individual Style of Activity of Preschool Children. Journal of New Medical Technologies, 21 (3), 143-146. doi: 10.12737/5921

[41] Balykin, M. V., Makhova, N. A., Britvina, N. D. (2009). Psychoemotional and functional changes in children with deviations on intellectual development after the course of faltering normobaric hypoxia. Journal of New Medical Technologies, 16 (3), 44-46.

[42] Semenza, G. L. (2000). Signal trasnsducktion to hypoxia-inducible factor 1. Genes \& Development, 14, 1983-1991.

[43] Prass, K., Scharff, A., Ruscher, K., Lowl, D., Muselmann, C., Victorov, I. et. al. (2003). Hypoxia-Induced Stroke Tolerance in the Mouse Is Mediated by Erythropoietin. Stroke, 34 (8), 1981-1986. doi: 10.1161/01.str.0000080381.76409.b2

[44] Zhan, L., Wang, T., Li, W., Xu, Z. C., Sun, W., Xu, E. (2010). Activation of Akt/FoxO signaling pathway contributes to induction of neuroprotection against transient global cerebral ischemia by hypoxic pre-conditioning in adult rats. Journal of Neurochemistry, 114 (3), 897-908. doi: 10.1111/j.14714159.2010.06816.x

[45] Jones, N. M., Bergeron, M. (2004). Hypoxia-induced ischemic tolerance in neonatal rat brain involves enhanced ERK1/2 signaling. Journal of Neurochemistry, 89 (1), 157-167. doi: 10.1111/j.14714159.2004.02324.x

[46] Ferrari, M., Jain, I. H., Goldberger, O., Rezoagli, E., Thoonen, R., Chen, K.-H. (2017). Hypoxia treatment reverses neurodegenerative disease in a mouse model of Leigh syndrome. Proceedings of the National Academy of Sciences, 114 (21), 4241-4250. doi: 10.1073/pnas.1621511114

[47] Lisukha, L. M. (2015). Effect of intermittent normobaric hypoxia on vegetative homeostasis and hemodynamic parameters of children aged 6-11 years who live at radiation contaminated territories. Sovremennaya pediatriya, 71 (7), 66-70. doi: 10.15574/sp.2015.71.66

[48] Lisukha, L. M., Berezovsky, V. Y. (2016). Influence of intermittent normobaric hypoxia on cardiovascular system and hemodynamic parameters of children lived at radiation contaminated territories. Fiziolohichnyi Zhurnal, 62 (4), 46-52. doi: 10.15407/fz62.04.046

[49] Stepanova, Ye. I., Berezovsky, V. Ya., Kolpacov, I. Ye., Kondrashova, V. H., Vdovenco, V. Yu., Lytvynets, O. M., Lisukha, L. M., et. al. (2016). Efficiency of endothelian dysfunction correction in children/ residents of radioactively contaminated areas using the method of intermittent normobaric hypoxi therapy. Problems of radiation medicine and radiobiology, 21, 322-335.

[50] Lisukha, L. (2017). Effect of intermittent normobaric hypoxia on somatic vegetative status of children who live at radioactively contaminated territories. Kyiv, 24. 